\title{
The application of intraoperative frozen section examination in multiparametric magnetic resonance imaging/transrectal ultrasound fusion prostate biopsy during a major pandemic
}

\author{
Xue-Fei Ding, Yang Luan, Liang-Yong Zhu, Qin Xiao, Ji Chen, Hao-Peng Chen, Yue-Xing Han, Zhong Liu \\ Clinical Medical College, Yangzhou University, Yangzhou, China
}

Contributions: (I) Conception and design: XF Ding, Y Luan; (II) Administrative support: XF Ding; (III) Provision of study materials or patients: XF Ding, Y Luan; (IV) Collection and assembly of data: Y Luan, LY Zhu, Q Xiao, J Chen, HP Chen, YX Han, Z Liu; (V) Data analysis and interpretation: Y Luan, LY Zhu; (VI) Manuscript writing: All authors; (VII) Final approval of manuscript: All authors.

Correspondence to: Yang Luan. Clinical Medical College, Yangzhou University, 98 West Nantong Road, Yangzhou 225001, China.

Email: Luan_Y@163.com.

Background: To investigate the value of intraoperative frozen section examination (IFSE) in multiparametric magnetic resonance imaging/transrectal ultrasound (mpMRI/TRUS) fusion prostate biopsy in a major pandemic.

Methods: A total of 35 patients were prospectively enrolled in our hospital from March 2020 to January 2021. The mpMRI/TRUS fusion system was used to perform a targeted biopsy, and the collected specimens were examined by IFSE (Observation Group 1). Then, a targeted biopsy was performed again for routine pathological examination (Observation Group 2). Finally, a systemic biopsy was performed, and the obtained specimens were routinely examined (Control Group). The positive rate, single core positive rate, Gleason score, and time to obtain pathological reports were compared between the groups.

Results: The positive rate was 48.6\% (17/35) in the control group, 48.6\% (17/35) in Observation Group 1 , and $51.4 \%$ (18/35) in Observation Group 2, showing no significant difference ( $\mathrm{P}>0.05)$. The single core positive rates were $17.8 \%, 44.6 \%$, and $47.1 \%$ in the Control Group, Observation Group 1, and Observation Group 2, respectively. Observation Group 1 and Observation Group 2 were significantly different from the Control Group $(\mathrm{P}<0.001)$. No participants in Observation Group 1 had increased or decreased Gleason scores compared with those in Observation Group 2. The time to obtain the pathological report was $0.025 \pm 0.014$ days and 4.216 \pm 1.073 days for Observation Group 1 and Observation Group 2, respectively, showing a significant difference $(\mathrm{P}<0.001)$.

Conclusions: This study showed that IFSE can not only rapidly obtain the pathological report of an mpMRI/TRUS biopsy, but can also ensure the accuracy of the pathological diagnosis.

Trial Registration: CHICTR, Identifier: ChiCTR2000040789. Registered 10 December 2020 Retrospectively registered, http://www.chictr.org.cn/edit.aspx?pid=63252\&htm=4.

Keywords: Frozen section; prostate cancer; biopsy; pandemic

Submitted May 02, 2021. Accepted for publication Dec 22, 2021; Published online: 17 Jan 2022.

doi: $10.21037 /$ qims-21-473

View this article at: https://dx.doi.org/10.21037/qims-21-473 


\section{Introduction}

Prostate cancer is a serious threat to men's health worldwide. According to the latest data from the American Institute of Cancer Research, prostate cancer is the most frequently diagnosed male malignancy (1). In China, prostate cancer has risen to being the sixth most prevalent cancer. The proportion of new prostate cancer patients in advanced stages is much higher than that in European and American countries, and the prognosis and overall survival (OS) rate are less favorable than those in European and American countries (2). Therefore, early diagnosis and effective treatment are key to improving the survival time and quality of life of prostate cancer patients (3). At present, pathological examination is still the gold standard for the diagnosis of prostate cancer.

Coronavirus disease 2019 (COVID-19) is characterized by rapid transmission, a wide infection area, and difficulties in prevention and control. Within a short period of time, the pandemic has escalated globally $(4,5)$. While actively preventing and controlling the pandemic and intercepting its spread, quickly obtaining the pathological results of prostate biopsies and reducing the hospital stay of patients to reduce their chance of close contact with a COVID-19 patient has presented a new challenge.

With the rapid development of the fields of accurate medical imaging technology and artificial intelligence, prostate biopsy has also made fresh progress. Current studies focus on improving the accuracy of single core biopsy; in other words, without an increase in the number of cores, targeted prostate biopsy is performed under the guidance of multiparametric magnetic resonance imaging (mpMRI) and transrectal ultrasound (TRUS) (6). The mpMRI/TRUS fusion prostate biopsy has shown superiority in improving the positive rate of using a single needle and the total positive rate: on the one hand, it reduces the number of cores needed, which reduces the pain experienced by patients, and, on the other hand, it effectively improves the accuracy of biopsy $(7,8)$.

Intraoperative frozen section examination (IFSE) can be performed without procedures such as tissue fixation, dehydration, transparency, and embedding, which reduces some intermediate links, and it can determine the presence and nature of lesions in a relatively short time, effectively assisting clinicians with decision making regarding surgical planning (9). Therefore, we attempted to directly perform IFSE on prostate specimens obtained by mpMRI/TRUS fusion biopsy, and to assess its feasibility and effectiveness.
We present the following article in accordance with the Strengthening the Reporting of Observational Studies in Epidemiology (STROBE) checklist (available at https://qims. amegroups.com/article/view/10.21037/qims-21-473/rc).

\section{Methods}

\section{Clinical data}

Patients who underwent mpMRI/TRUS biopsy at Clinical Medical College, Yangzhou University, from March 2020 to January 2021 were prospectively enrolled. The inclusion criteria were as follows: (I) patients with findings of nodules on digital rectal examination, (II) patients with mpMRI findings indicative of prostate cancer [Prostate Imaging Reporting and Data System v2 (PI-RADS V2) scores $\geq 3$ ], (III) patients with prostate-specific antigen (PSA) concentrations of $4-10 \mathrm{ng} / \mathrm{mL}$ with an abnormal free/ total PSA or PSA density, and (IV) patients with a PSA concentration $>10 \mathrm{ng} / \mathrm{mL}$. The exclusion criteria were as follows: (I) an MRI contraindication, (II) a poor MRI image quality or an incomplete image unable to be analyzed, and (III) mpMRI images revealing no suspicious areas. The study was conducted in accordance with the Declaration of Helsinki (as revised in 2013). This study was approved by the Ethics Committee of Clinical Medical College, Yangzhou University (No. 2020KY-013), and all participants provided their written informed consent.

All participants underwent mpMRI with a 3.0-T MR scanner (Signa HDxt, GE Medical Systems, Milwaukee, WI, USA) using a 32-channel, phase-controlled, front loop before the mpMRI/TRUS biopsy. While unaware of the patient's characteristics, 2 experienced radiologists read all the MRI scans.

The mpMRI images of all participants were scored in accordance with the PI-RADS V2 by 2 experienced radiologists who were blinded to the participants' prior clinical information (10), and a consensus was reached between them.

\section{Biopsy procedure}

The mpMRI/TRUS biopsy system (MIM Symphony, MIM Software, Cleveland, OH, USA) was used for all prostate biopsies. The system fused the TRUS and $m p M R I$ images in real-time to guide the biopsy needle through the transperineal approach. Firstly, we introduced the original data from mpMRI in the Digital 


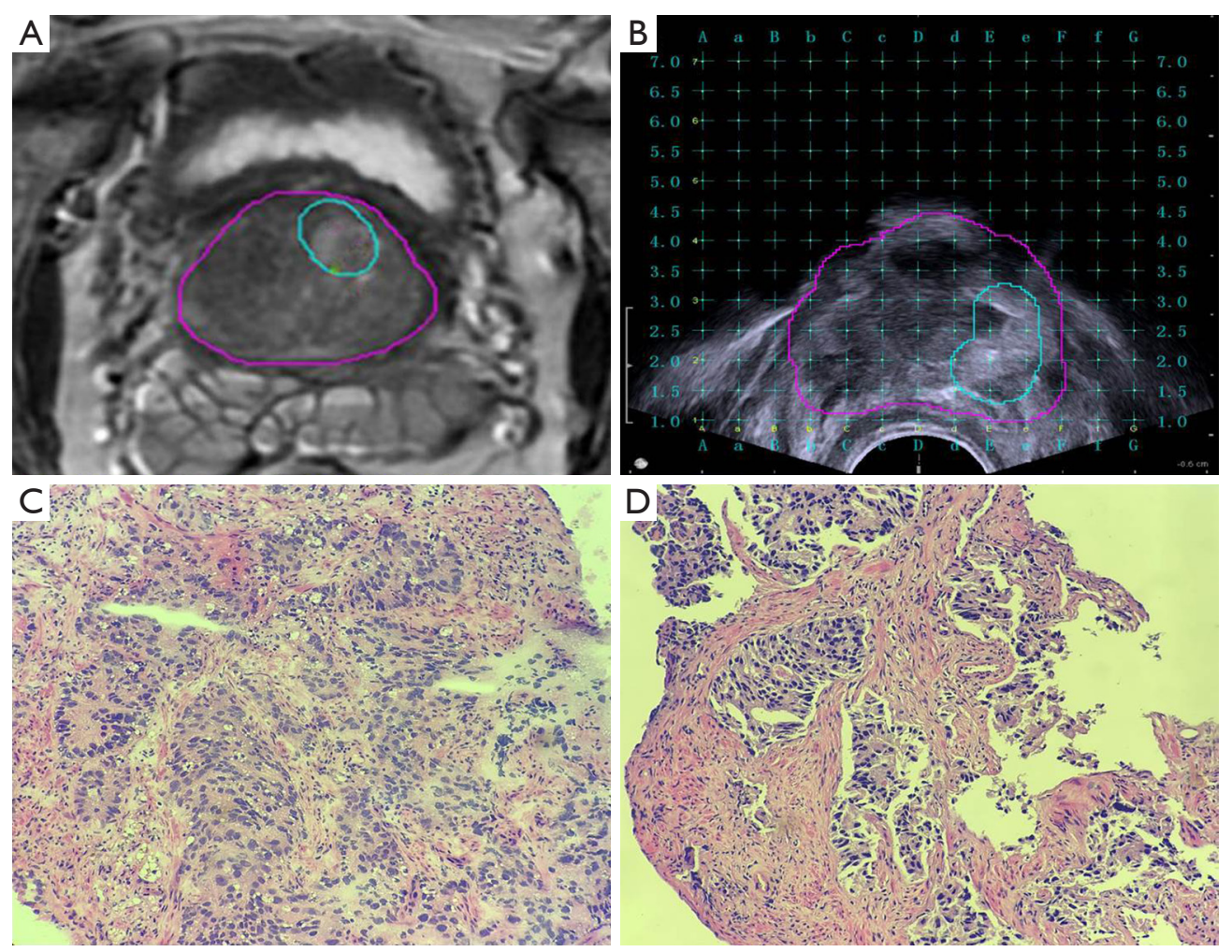

Figure 1 Images of the mpMRI/TRUS and the pathology. (A) Referring to multiple sequences of mpMRI, the regions of interest were delineated on T2-weighted imaging. The prostate target is contoured in purple. The suspected area of the prostate cancer target is contoured in blue; (B) the regions of interest delineated on mpMRI are fused onto the TRUS image. Targeted biopsy was performed under the guidance of mpMRI/TRUS image fusion; (C) image of intraoperative frozen section examination ( $\times 200)$; (D) image of routine pathological examination $(\times 200)$. mpMRI/TRUS, multiparametric magnetic resonance imaging/transrectal ultrasound.

Imaging and Communications in Medicine (DICOM) format to the image fusion system. Then, the suspicious areas were marked on the $\mathrm{T} 2$-weighted image (T2WI) (Figure 1A). Secondly, we placed the virtual probe in the rectum approximately $3 \mathrm{~mm}$ from the posterior wall of the prostate. Next, we adjusted the angle of the probe until the base plane aligned with the underlying prostate, and then adjusted the virtual grid to ensure that the prostate was in the proper position. Finally, we inserted the biplanar TRUS probe (Flex Focus 1202, BK Ultrasound Systems, Naerum, Denmark) into the patient's rectum. The fiducial landmark for registering the MRI and ultrasound images was represented by the prostate base. The reconstructed MRI axial images and the TRUS axial image were synchronously displayed on the same monitor. Morphological MRI data, including the suspicious areas, were superimposed on the TRUS images in real time to guide the biopsy needle (Figure 1B).
Template-guided biopsy with 2-3 cores was performed on the suspected lesions, and an IFSE was performed on the obtained specimens (Observation Group 1) (Figure 1C). Then, template-guided biopsy with $2-3$ cores was again performed on the suspected lesions, and a routine pathological examination was performed on the obtained specimens (Observation Group 2) (Figure 1D). Systematic biopsy was performed after the mpMRI/TRUS biopsy, and a routine pathological examination of the specimens was performed (Control Group).

The specimens were placed in the optimal cutting temperature compound and frozen at $-20{ }^{\circ} \mathrm{C}$ for pretreatment. Then, the specimens were cut into $5 \mu \mathrm{m}$ slices with a constant temperature freezing slicer. Finally, the slices were stained (hematoxylin-eosin staining) and sealed. All prostate biopsy specimens were reviewed by 2 experienced pathologists who were blinded to the participants' MRI and TRUS results. 


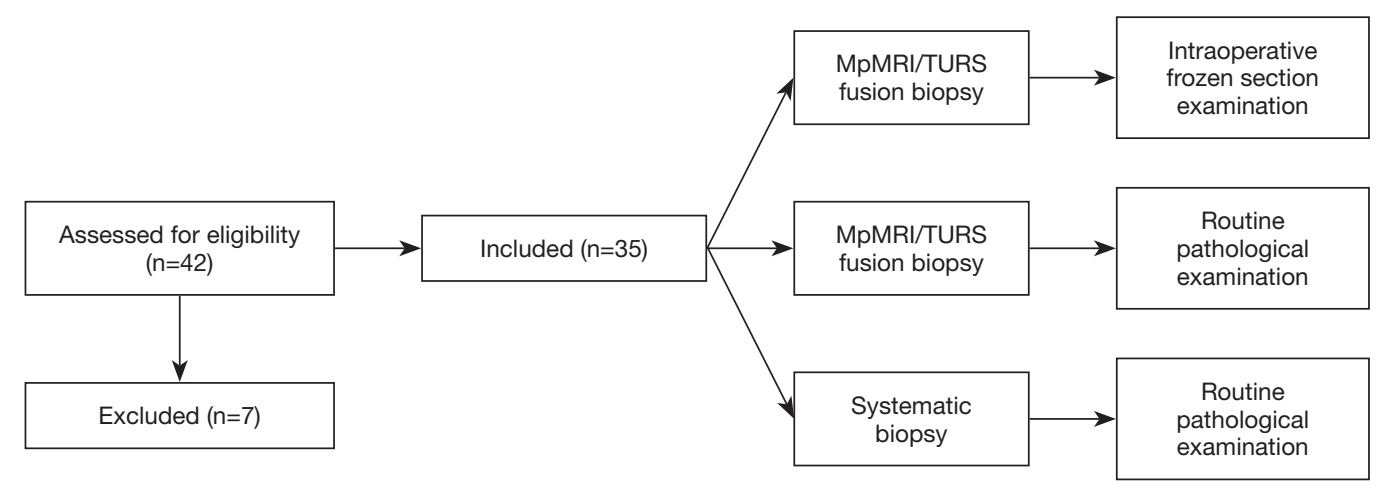

Figure 2 The flow diagram of the study.

\section{Statistical analysis}

The SPSS 19.0 statistical software program (IBM Corp., Armonk, NY, USA) was used to process the data. Mean $\pm \mathrm{SD}$ was used to describe quantitative statistics, and the independent sample $t$-test (normal distribution) or the Mann-Whitney $\mathrm{U}$ test (deviated distribution) were used to compare the intergroup differences. In addition, the rates were compared using the chi-square $\left(\chi^{2}\right)$ test. A value of $\mathrm{P}<0.05$ was considered statistically significant.

\section{Results}

A total of 35 patients were included; the average age of the participants was $69.3 \pm 7.8$ years, and the average PSA concentration was $14.5 \pm 6.8 \mu \mathrm{g} / \mathrm{L}$ (Figure 2). Of the 35 participants, $20(57.1 \%)$ were diagnosed with prostate cancer. The positive rate of the Control Group was 48.6\% (17/35), and the positive rate of Observation Group 2 was $51.4 \%(18 / 35)$. There was no significant difference in the positive rate between the 2 groups $(\mathrm{P}=0.811)$. In the control group, a total of 437 cores were biopsied, of which 78 were positive, and the positive rate of a single core was $17.8 \%$. In Observation Group 2, a total of 87 cores were biopsied, of which 41 were positive, and the positive rate of a single core was $47.1 \%$. The positive rate of a single core was significantly different between the 2 groups $(\mathrm{P}<0.001)$. In the control group, the Gleason score ranged from 6 to 9 , with an average of $7.54 \pm 1.05$. The rates of Gleason scores 6,7 , and $\geq 8$ were $23.5 \%$ (4/17), $47.1 \%$ (8/17), and $29.4 \%$ $(5 / 17)$, respectively. The Gleason score of Observation Group 2 was 6 to 10 , with an average of $7.81 \pm 1.12$. The proportions of Gleason scores 6,7 , and $\geq 8$ were $16.7 \%$ (3/18), 50\% (9/18), and 33.3\% (6/18), respectively. There was no significant difference in the Gleason scores between the control group and Observation Group $2(\mathrm{P}=0.302)$. However, among the 13 patients with prostate cancer who were diagnosed in both the Control Group and Observation Group 2, 4 participants in Observation Group 2 had higher Gleason scores than those in the Control Group.

The positive rate of Observation Group 1 was 48.6\% (17/35), with a total of 83 cores, of which 37 were positive, and the positive rate of a single core was $44.6 \%$. There were no significant differences in the positive rate or single core positive rate between Observation Group 1 and Observation Group $2(\mathrm{P}>0.05)$. The Gleason score in Observation Group 1 was 6 to 10, with an average of $7.68 \pm 1.31$, showing no significant difference when compared with Observation Group $2(\mathrm{P}=0.433)$. In Observation Group 1, 17.6\% (3/17), $52.9 \%(9 / 17)$, and $29.4 \%(5 / 17)$ of the participants had Gleason scores of 6,7 , and $\geq 8$, respectively. Of the 16 participants with prostate cancer detected in both Observation Group 1 and Observation Group 2, none of the participants showed an increase or decrease in the Gleason score. A single participant in Observation Group 1 was diagnosed with prostate cancer, but not in Observation Group 2. Meanwhile, prostate cancer was diagnosed in 2 participants in Observation Group 2, but not in Observation Group 1.

The time to obtain the pathological reports for patients in Observation Group 1 was $0.025 \pm 0.014$ days. The time to obtain the pathological reports for patients in Observation Group 2 was $4.216 \pm 1.073$ days, and there was a significant difference between the 2 groups $(\mathrm{P}<0.001)$.

\section{Discussion}

In December 2019, a novel coronavirus appeared in Wuhan, China. It was named COVID-19, and it spread 
rapidly throughout China and the world. In a major pandemic such as that of COVID-19, patients with highly suspected prostate cancer need to undergo accurate biopsy and obtain pathological results quickly to effectively reduce their hospital stay. In addition, early pathological results can be obtained, and according to whether the pathological results show benign or malignant cancer, the corresponding treatment can be initiated as soon as possible to improve the prognostic effect. In addition, treating prostate cancer in its early stages can help patients alleviate the heavy burden of "cancer fear" and improve their quality of life. Therefore, it is necessary for doctors and patients to identify the pathological characteristics of prostate nodules as soon as possible after their discovery.

The MRI can accurately locate suspected lesions of the prostate. Guided by MRI, a targeted prostate biopsy can be performed. An mpMRI/TRUS biopsy combines the advantages of the high sensitivity and specificity of the MRI lesion display and the real-time display and flexibility of TRUS, which, to a certain extent, makes up for the deficiency of targeted biopsy guided by MRI in real time $(7,11)$. The equipment does not need to be changed and patients do not need to change position. Additional targeted biopsies can be performed on suspicious lesions shown by mpMRI, which is relatively simple and easy to operate.

The IFSE is a method of quickly freezing specimens to a certain grade of hardness at low temperatures and then slicing the specimens. Compared with routine pathological examination, IFSE does not need to fix, dehydrate, or embed the specimens, which saves operational time and is quick and simple. In the process of rapid freezing, various antigenic activities and enzymes can be well preserved, especially organic solvents and those with poor tolerance to temperature, which reduces the variation in tissue structure and improves the accuracy of testing. At present, IFSE is widely used in breast, lung, uterine, thyroid, and other tissues, and provides a reliable basis for pathological diagnosis (12-15). Hence, IFSE results should be used to guide the surgical method of prostate nodules, such as that of breast nodules, to avoid patient suffering caused by multiple surgeries and to reduce the cost of treatment.

Therefore, beginning in March 2020, we applied IFSE in mpMRI/TRUS biopsy to explore its effectiveness and reliability. In this study, the positive rate of Observation Group 2 was $51.4 \%$ and the positive rate of the control group was $48.6 \%$. There was no significant difference in the positive rate between the 2 groups $(\mathrm{P}=0.811)$; however, the positive rate of a single core in Observation Group 2 was 2.65 times $(47.1 \% 17.8 \%)$ that of the control group. Wang et al. (16) performed a total of 2,300 cores in 168 patients, among which the single core positive rate of MRI/ TRUS biopsy was $51.76 \%$ higher than that of systematic biopsy (19.64\%). Otherwise, the average Gleason score of Observation Group 2 was $7.81 \pm 1.12$ and the average Gleason score of the control group was $7.54 \pm 1.05$. Among the 13 patients who were diagnosed with prostate cancer by both systematic biopsy and MRI/TRUS biopsy, the Gleason score of the 4 patients diagnosed by MRI/TRUS biopsy was higher than that of patients diagnosed by systematic biopsy. Siddiqui et al. (17) performed a 12-core systemic biopsy and MRI/TRUS biopsy in 582 patients, and showed an increase in the Gleason score in 32\% of the patients diagnosed via MRI/TRUS biopsy. Therefore, compared with systematic biopsy, MRI/TRUS biopsy can achieve a similar positive rate but can significantly improve the single core positive rate and avoid missed diagnoses of high-risk prostate cancer.

Further comparison between Observation Group 1 and Observation Group 2 showed no significant differences in the positive rate, single core positive rate or Gleason score $(\mathrm{P}>0.05)$, and there was no decrease or improvement in the Gleason score between the 2 groups. More importantly, the study showed that the time for obtaining pathological results in Observation Group 1 was significantly shorter than that in Observation Group 2. This not only greatly reduces the patients' length of hospital stay and reduces the rate of infection caused by the pandemic due to exposure in the hospital, but also shortens the time of the "cancer fear" period while patients are waiting for their pathological results. Further, we tried to immediately perform radical prostatectomy for patients whose IFSE results confirmed prostate cancer, which has achieved better treatment results, reduced hospitalization times and length of stay, and reduced hospitalization costs.

In addition, 1 and 2 patients were diagnosed with prostate cancer in Observation Group 1 and Observation Group 2, respectively, but not in Observation Group 2 and Observation Group 1, respectively. The possible reasons were the heterogeneity of the lesion, the difference in the quality of the biopsy specimen, or the subjective influence of the pathologist. Therefore, further research is needed.

There were several limitations in this study: (I) this study did not systematically compare biopsy pathology with radical pathology, nor did it compare biopsy pathology with postoperative whole-mount sections. Based on the 
technique, we will next explore whether IFSE can accurately describe the spatial distribution of prostate cancer; (II) the number of patients included in this study was still relatively small, and further data is required; (III) patients with no suspicious lesions on mpMRI images were not included in this study, which may have led to selection bias.

In summary, IFSE can quickly obtain the pathological results of mpMRI/TRUS biopsy and has a high accuracy, which is worthy of promotion and application in a major pandemic.

\section{Acknowledgments}

Funding: This work was supported by the Yangzhou Science and Technology Plan Project (No. YZ 2019053).

\section{Footnote}

Reporting Checklist: The authors have completed the Strengthening the Reporting of Observational Studies in Epidemiology (STROBE) checklist. Available at https://qims. amegroups.com/article/view/10.21037/qims-21-473/rc

Conflicts of Interest: All authors have completed the ICMJE uniform disclosure form (available at https://qims. amegroups.com/article/view/10.21037/qims-21-473/coif). The authors have no conflicts of interest to declare.

Ethical Statement: The authors are accountable for all aspects of the work in ensuring that questions related to the accuracy or integrity of any part of the work are appropriately investigated and resolved. The study was conducted in accordance with the Declaration of Helsinki (as revised in 2013). This study was approved by the Ethics Committee of Clinical Medical College, Yangzhou University (No. 2020KY-013), and all participants signed informed consent forms.

Open Access Statement: This is an Open Access article distributed in accordance with the Creative Commons Attribution-NonCommercial-NoDerivs 4.0 International License (CC BY-NC-ND 4.0), which permits the noncommercial replication and distribution of the article with the strict proviso that no changes or edits are made and the original work is properly cited (including links to both the formal publication through the relevant DOI and the license). See: https://creativecommons.org/licenses/by-nc-nd/4.0/.

\section{References}

1. Siegel RL, Miller KD, Jemal A. Cancer statistics, 2019. CA Cancer J Clin 2019;69:7-34.

2. Chen W, Sun K, Zheng R, Zeng H, Zhang S, Xia C, Yang $\mathrm{Z}, \mathrm{Li} \mathrm{H}, \mathrm{Zou} \mathrm{X}, \mathrm{He} \mathrm{J}$. Cancer incidence and mortality in China, 2014. Chin J Cancer Res 2018;30:1-12.

3. Heijnsdijk EA, Wever EM, Auvinen A, Hugosson J, Ciatto S, Nelen V, et al. Quality-of-life effects of prostate-specific antigen screening. N Engl J Med 2012;367:595-605.

4. Huang C, Wang Y, Li X, Ren L, Zhao J, Hu Y, et al. Clinical features of patients infected with 2019 novel coronavirus in Wuhan, China. Lancet 2020;395:497-506.

5. Zhu N, Zhang D, Wang W, Li X, Yang B, Song J, Zhao X, Huang B, Shi W, Lu R, Niu P, Zhan F, Ma X, Wang D, Xu W, Wu G, Gao GF, Tan W; China Novel Coronavirus Investigating and Research Team. A Novel Coronavirus from Patients with Pneumonia in China, 2019. N Engl J Med 2020;382:727-33.

6. Baccaglini W, Glina FPA, Pazeto CL, Bernardo WM, Sanchez-Salas R. mpMRI-targeted biopsy versus systematic biopsy for clinically significant prostate cancer diagnosis: a systematic review and metaanalysis. Curr Opin Urol 2020;30:711-9.

7. Drost FH, Osses D, Nieboer D, Bangma CH, Steyerberg EW, Roobol MJ, Schoots IG. Prostate Magnetic Resonance Imaging, with or Without Magnetic Resonance Imaging-targeted Biopsy, and Systematic Biopsy for Detecting Prostate Cancer: A Cochrane Systematic Review and Meta-analysis. Eur Urol 2020;77:78-94.

8. Wegelin O, van Melick HHE, Hooft L, Bosch JLHR, Reitsma HB, Barentsz JO, Somford DM. Comparing Three Different Techniques for Magnetic Resonance Imaging-targeted Prostate Biopsies: A Systematic Review of In-bore versus Magnetic Resonance Imaging-transrectal Ultrasound fusion versus Cognitive Registration. Is There a Preferred Technique? Eur Urol 2017;71:517-31.

9. Cipolla C, Graceffa G, Cabibi D, Gangi G, Latteri M, Valerio MR, Vieni S. Current Role of Intraoperative Frozen Section Examination of Sentinel Lymph Node in Early Breast Cancer. Anticancer Res 2020;40:1711-7.

10. Park SY, Jung DC, Oh YT, Cho NH, Choi YD, Rha KH, Hong SJ, Han K. Prostate Cancer: PI-RADS Version 2 Helps Preoperatively Predict Clinically Significant Cancers. Radiology 2016;280:108-16.

11. Ding XF, Luan Y, Wang F, Xu YZ, Guo CH, Zhu LY. The application of a targeted periprostatic nerve block in transperineal template-guided prostate biopsies. Quant 
Imaging Med Surg 2020;10:2125-32.

12. Tamanuki T, Namura M, Aoyagi T, Shimizu S, Suwa T, Matsuzaki H. Effect of Intraoperative Imprint Cytology Followed by Frozen Section on Margin Assessment in Breast-Conserving Surgery. Ann Surg Oncol 2021;28:1338-46.

13. Gagné A, Racine É, Orain M, Meziou S, Simard S, Couture C, Pagé S, Trahan S, Ugalde P, Lacasse Y, Joubert D, Joubert P. Identification of Grossing Criteria for Intraoperative Evaluation by Frozen Section of Lung Cancer Resection Margins. Am J Surg Pathol 2018;42:1495-502.

14. Balaya V, Guani B, Benoit L, Magaud L, Bonsang-Kitzis H, Ngô C, Le Frère-Belda MA, Mathevet P, Lécuru F. Diagnostic value of frozen section examination of sentinel lymph nodes in early-stage cervical cancer at the time of ultrastaging. Gynecol Oncol 2020;158:576-83.

Cite this article as: Ding XF, Luan Y, Zhu LY, Xiao Q, Chen J, Chen HP, Han YX, Liu Z. The application of intraoperative frozen section examination in multiparametric magnetic resonance imaging/transrectal ultrasound fusion prostate biopsy during a major pandemic. Quant Imaging Med Surg 2022;12(4):2378-2384. doi: 10.21037/qims-21-473
15. Mallick R, Stevens TM, Winokur TS, Asban A, Wang TN, Lindeman BM, Porterfield JR, Chen H. Is FrozenSection Analysis During Thyroid Operation Useful in the Era of Molecular Testing? J Am Coll Surg 2019;228:474-9.

16. Wang XM, Bao J, Hu CH, Zhang ZS, Xi QL, Hou JQ. The value of multiparametric MRI and transrectal ultrasound fusion guided biopsy in the detection of clinical significant prostate cancer. Chinese Journal of Radiology 2020;054:215-20.

17. Siddiqui MM, Rais-Bahrami S, Truong H, Stamatakis L, Vourganti S, Nix J, Hoang AN, Walton-Diaz A, Shuch B, Weintraub M, Kruecker J, Amalou H, Turkbey B, Merino MJ, Choyke PL, Wood BJ, Pinto PA. Magnetic resonance imaging/ultrasound-fusion biopsy significantly upgrades prostate cancer versus systematic 12-core transrectal ultrasound biopsy. Eur Urol 2013;64:713-9. 